\title{
Sizing gold nanoparticles by optical extinction spectroscopy
}

\author{
L B Scaffardi ${ }^{1,3}$, N Pellegri ${ }^{2}$, O de Sanctis $^{2}$ and J O Tocho ${ }^{1}$ \\ ${ }^{1}$ Facultad de Ciencias Exactas, UNLP and Centro de Investigaciones Ópticas (CIOp), \\ CC124, 1900 La Plata, Argentina \\ ${ }^{2}$ Laboratorio de Materiales Cerámicos, FCElyA, IFIR, UNR, Rosario, Argentina \\ E-mail: lucias@ciop.unlp.edu.ar
}

Received 30 August 2004, in final form 9 November 2004

Published 10 December 2004

Online at stacks.iop.org/Nano/16/158

\begin{abstract}
The measurement of optical extinction is used to determine the size of nearly spherical gold nanoparticles suspended in solution, produced by a 'reverse micelles' process. The contrast between the maximum and the minimum in the extinction spectra around 450 and $520 \mathrm{~nm}$ shows a linear dependence with the mean radius of the gold particles less than $3 \mathrm{~nm}$; however, the method can be used to size particles up to $7 \mathrm{~nm}$. Experimental results for extinction spectra can be fitted by Mie's theory if the optical constants from bulk material values are modified by introducing the limitation of the mean free path due to collisions of conduction electrons with the boundary of the nanoparticles.
\end{abstract}

\section{Introduction}

The determination of particle size in the micron and submicron region has been a subject of increasing interest during recent years. Among the diverse fields of applied sciences that involve this subject, the studies of atmospheric and combustion aerosols, the analysis of interstellar dust, and the characterization of many biological, chemical and physical systems of interest for industrial processes stand out. Materials science is another field of interest where it is recognized that many properties of materials are affected by the size and characteristics of the particles within them. To understand the influence of particles in nature or to control industrial processes involving them it is essential to develop methods to measure their size [1]. Many physical techniques are available, but optical methods are often preferred because they can be non-disturbing, the measurements can be made remotely, and analysis can be very rapid. The optical methods include static and dynamic dispersion; lidar [2,3]; counters, where the size is correlated with the height of the pulse of light dispersed by the particle; extinction spectrum; and many others. Among them, the spectral extinction method is one of most used because it requires a simple optical disposition like the slight adaptation of a commercial spectrophotometer and it can work in an extended interval of sizes.

3 Author to whom any correspondence should be addressed.
The spectral extinction method can be easily performed for particles in diluted liquid suspensions. The knowledge of the refractive index for both the particle material and the solvent, as a function of the wavelength, is necessary. As usual, experimental results are fitted by Mie calculations for spherical particles. This method has proved to be very useful for sizing particles in the submicron range, where it can be compared with both transmission electronic microscopy (TEM) and scanning electronic microscopy (SEM) [4]. To deal with the distribution of radius, extinction measurements at a discrete set of wavelengths are performed (multispectral extinction). Inversion of the experimental results to get the distribution of sizes is not a trivial task, but it can be solved by a nonlinear inversion algorithm [5]. As is common for all the optical methods, the range of the radius determined for optical extinction is related to the range of the wavelength used. As an example, when a commercial spectrophotometer with a wavelength range of $300-1100 \mathrm{~nm}$ is used, particle sizing over a radius range of $300-1400 \mathrm{~nm}$ can be attained.

In particular, measurements of optical extinction as a function of wavelength offer useful information of particle size on the micrometre range. This analysis yields, in general, inaccurate results when the sizes of particles are much smaller than the wavelength, thus making it an unsuitable method to measure particles with radius below $100 \mathrm{~nm}$.

Metal particles constitute one important exception to this rule because the extinction spectra present clear size effects 
for radius well below the wavelength. For this reason colloidal solutions of noble metals, like copper, gold and silver, present intense colour that is absent in bulk material. For spherical particles Mie's theory predicts one strong absorbance for small particles at the frequency where $\varepsilon=-\left[2+(12 / 5) x^{2}+\cdots\right] \varepsilon_{m}$. Here $\varepsilon$ is the complex dielectric function for the particle, $\varepsilon_{m}$ is the dielectric function for the solvent, and $x$ is the size parameter [6]. For vanishing small particles (Rayleigh limit) surface resonance modes can be achieved, and strong extinction is observed at the Fröhlich frequency where $\varepsilon=$ $-2 \varepsilon_{m}$. The size effect on the extinction spectra is explained because, in general, the real part of the dielectric function $\left(\varepsilon^{\prime}\right)$ is an increasing function of the frequency, and when $x$ increases, the absorption maximum is shifted to longer wavelength. This behaviour is used extensively in sizing metal particles, and is known as the extrinsic size effect on the plasmon band absorption [7]. In particular for gold particles, this method can be applied for a radius in the range $10-100 \mathrm{~nm}$. Below $10 \mathrm{~nm}$, the absorption spectrum peaks near $520 \mathrm{~nm}$ become independent of size, except for a varying intensity due to the dependence between the absorption cross section and the volume of the particle.

For small enough metal particles, well in the nanometre region, the extinction spectra show a new size dependence that can be used to characterize the radius. This region, known as the intrinsic size effect, can be studied by assuming that the dielectric function of the bulk must be modified for nanoparticles. The present work deals with gold particles whose radii are included in this region. These particles are used in the development of materials that have acquired great importance during recent years in different areas of scientific research and nanotechnology.

Many of the structures within the human body, such as proteins, are measured in nanometres, making nanotechnology an alluring area of biomedical research. The growing ability of researchers to synthesize nanomaterials opens up the possibility of using synthetic molecules such as biosensors to probe cellular functions. In the optical field, semiconductor and metal nanoparticles are of interest due to their many important applications in the development of optical devices $[8,9]$. In the area of semiconductor technology, the band gap of semiconductor nanoparticles can be controlled by the quantum size effect. In addition, it has been reported that the band structure of Si with indirect transition can be changed into that of direct transition by down-sizing of nanoparticles.

Many potential industrial applications of nanoparticles, such as quantum dots and tuneable laser diodes, require extreme monodispersity of particles. The generation of monodisperse nanoparticles is also important for nanoparticle instrumentation development and calibration. The abovementioned research and development areas need the knowledge of sizing distribution.

Below $10 \mathrm{~nm}$, sizing effects over the dielectric functions open a new window for measurements. This work shows a way to determinate the size of gold nanoparticles in this nanometric region. The theoretical fit of the extinction curves was performed using Mie theory, taking into account a modification of the bulk dielectric function for the case of very small particles.

Extinction measurements with a commercial spectrophotometer can be an altemative simple and economical technique when special electronic microscopy (TEM or SEM) is not available. Recently the possibility to size nanoparticles down to $1 \mathrm{~nm}$ by dynamic light scattering techniques was claimed by some manufacturers.

As with other sizing techniques, optical extinction spectroscopy cannot be considered as an absolute or universal method. This work is related to mean size determinations, and no distribution of size was considered. This is an important limitation when it is compared with microscopic measurements that allow mean size and distribution results simultaneously.

\section{Experimental section}

The gold nanoparticles analysed were synthesized in 'reverse micelles' in water-hydrocarbon phases and isolated with a stabilizer. The solvent was n-heptane and the water was triply distilled. Hydrogen tetrachloroaurate (III) hydrate $\left(\mathrm{HAuCl}_{4} \cdot 3 \mathrm{H}_{2} \mathrm{O}\right)$ (Aldrich, 25416) was selected as the source of gold. Sodium bis (2-ethylhexyl) sulfosuccinate (AOT, Sigma) was used as surfactant and $\mathrm{N}$-[3(trimethoxysilyl)propyl]diethylenetriamine (Aldrich, 41 334, hereafter ATS) as stabilization agent. The $\left[\mathrm{H}_{2} \mathrm{O}\right] /[\mathrm{AOT}]$ ratio was equal to 6 , and it was maintained constant for all samples. The 'pool' size of the 'reverse micelles' was changed by using values of 6,10 and 20 for the [ATS]/[ $\left.\mathrm{Au}^{3+}\right]$ ratio. The gold nanoparticles were formed by photoreduction of the $\mathrm{HAuCl}_{4}$ irradiating the $\mathrm{Au}^{3+}$ micellar solutions with light emitted from a mercury high-pressure lamp (maximum intensity at $\lambda=365 \mathrm{~nm}, 400 \mathrm{~W}$ ). As the photochemistry of gold nanoparticles in reverse micelles depends strongly on the synthesis conditions, experimental conditions were developed such that all samples received the same amount of radiation. In order to remove the nanoparticles from the micellar solution, a water-acetone mixture (3:1 volume ratio) was added to the $\mathrm{Au}$ particle micellar solution under strong stirring. The micellar structure broke down and a two-phase separation took place; the upper phase contained the gold nanoparticles dispersed in heptane and the lower phase contained acetone, water and AOT. The nanoparticles were obtained by evaporating the heptane at $70^{\circ} \mathrm{C}$ under a pressure of $5 \mathrm{kPa}$. More details of the preparation of the particles can be found in [10].

There is strong evidence that the particles are capped with the stabilization agent ATS and that this helps to maintain the gold particles in the dispersion state in organic solvents after extraction from that synthesis medium. For this reason samples were dispersed in heptane for the UV-visible extinction measurements. THE Spectra were recorded using a Beckman Spectrophotometer (Model 600), using n-heptane as the reference background. The optical density for $1 \mathrm{~cm}$ light pass was kept below one to avoid multiple scattering effects. Experimental refractive index values of heptane for 434,486 , 589 and $656 \mathrm{~nm}$ were used [11] to calculate values for other wavelengths with a Sellmeier's dispersion equation.

Transmission electron microscopy (TEM, JEOL 1010, at $80 \mathrm{kV}$ ) images were obtained by application of a droplet of the nanoparticle-dispersed heptane solution on carbon coated copper grids. Figure 1 shows TEM images of three samples of gold nanoparticles, ATS-6, ATS-10 and ATS-20. The nanoparticle shape is nearly spherical, but some superposition arising from the sample preparation process is observed in the 

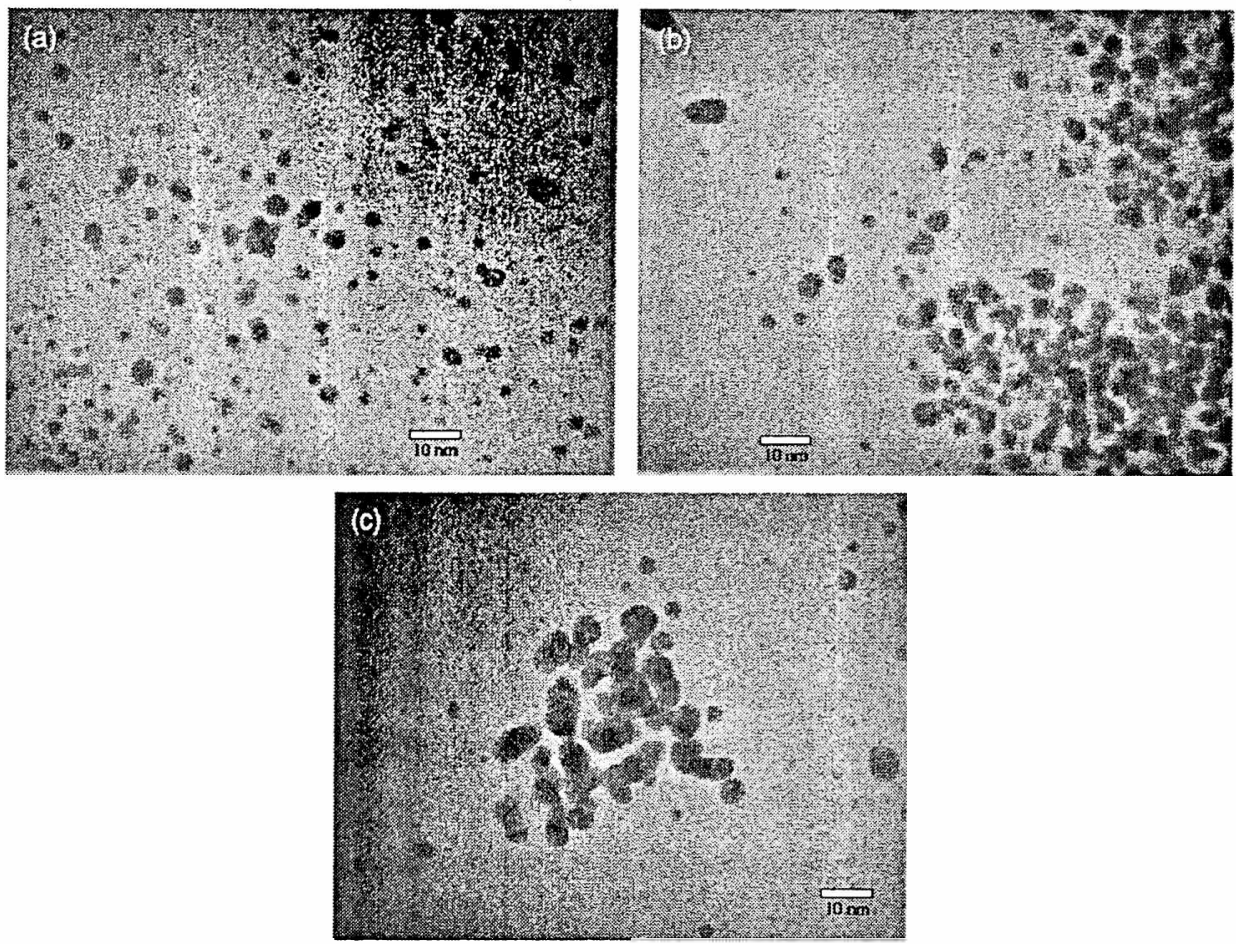

Figure 1. TEM images of (a) ATS-6, (b) ATS-10 and (c) ATS-20 gold nanoparticles samples. From histograms of these images the mean radius was determined as $1.34,1.58$ and $1.96 \mathrm{~nm}$, respectively.

pictures. The mean radius can be determined from statistical analysis from histograms of these pictures, discarding obvious superpositions. As expected, the particle size increased as the amount of stabilizer used in the preparation was increased.

\section{Theory and discussion}

The optical properties of materials are often described by two sets of quantities: the real and imaginary parts of the complex refractive index, $N=n+\mathrm{i} k$, and the real and imaginary parts of the complex dielectric function, $\varepsilon=\varepsilon^{\prime}+\mathrm{i} \varepsilon^{\prime \prime}$. These sets are not independent, since they are related by

$$
\begin{gathered}
\varepsilon^{\prime}=n^{2}-k^{2}, \\
\varepsilon^{\prime \prime}=2 n k .
\end{gathered}
$$

The optical properties for gold used in this work were taken or calculated from experimental values for $n$ and $k$ given by Palik [12]. For real metals where there is a substantial bound electron component, the dielectric function for the bulk can be decomposed into two terms, a free-electron term and an interband, or bound-electron term [6]. The dielectric function is additive, and it can be written as

$$
\varepsilon_{\text {bulk }}(\omega)=\varepsilon_{\text {bound-electrons }}(\omega)+1-\frac{\omega_{p}^{2}}{\omega^{2}+i \omega \gamma_{\text {bulk }}},
$$

where $\omega_{\mathrm{p}}$ is the bulk plasma frequency and $\gamma_{\text {bulk }}$ is the damping constant in the Drude model. From equation ( 3$), \varepsilon_{\text {bound-electron }}$ can be calculated by subtracting the free-electron part from $\varepsilon_{\text {bulk. }}$. Values for $\omega_{\mathrm{p}}=1.3 \times 10^{16} \mathrm{~s}^{-1}$ and $\gamma_{\text {bulk }}=1.64 \times$ $10^{14} \mathrm{~s}^{-1}$ were taken from [13].
For small particles, the plasma frequency is assumed to be independent of the size, while the damping, related to the mean free path for electrons, is strongly affected by the size. In particles smaller than the mean free path of conduction electrons in the bulk metal, the mean free path can be dominated by collisions with the particle boundary [14-16]. The mean free path limitation has been discussed by Kreibig and von Fragstein [14], who considered that the damping constant (the inverse of the collision time for conduction electrons in the Drude theory) is increased due to additional collisions with the boundary of the particle, and it can be written as

$$
\gamma(r)=\gamma_{\text {bulk }}+\frac{v_{\mathrm{F}}}{L}=\gamma_{\text {bulk }}+C \frac{v_{\mathrm{F}}}{r},
$$

where $v_{\mathrm{F}}$ is the electron velocity at the Fermi surface, and $L$ is the effective mean free path for collisions with the boundary. The value $v_{\mathrm{F}}=14.1 \times 10^{14} \mathrm{~nm} \mathrm{~s}^{-1}$ was used [13]. There is slight disagreement among various authors about the proportionality constant $C$ between $L$ and the radius $r$ of the particle. $C$ is a constant that includes details of the scattering processes. For isotropic electron scattering $C=1$ was calculated theoretically; for diffuse scattering its value is reduced to 0.75 , while for additional limitations due to the internal grain boundary $C$ can be greater than 1 [17].

Now, the real and the imaginary part of the dielectric function for a particle of radius $r$, take the form

$$
\begin{aligned}
& \varepsilon_{\text {size }}^{\prime}(\omega, r)=1-\frac{\omega_{p}^{2}}{\omega^{2}+\gamma^{2}(r)}+\varepsilon_{\text {bound-electron }}^{\prime}, \\
& \varepsilon_{\text {size }}^{\prime \prime}(\omega, r)=\frac{\gamma(r) \omega_{p}^{2}}{\left(\omega^{2}+\gamma^{2}(r)\right) \omega}+\varepsilon_{\text {bound-electron }}^{\prime \prime},
\end{aligned}
$$



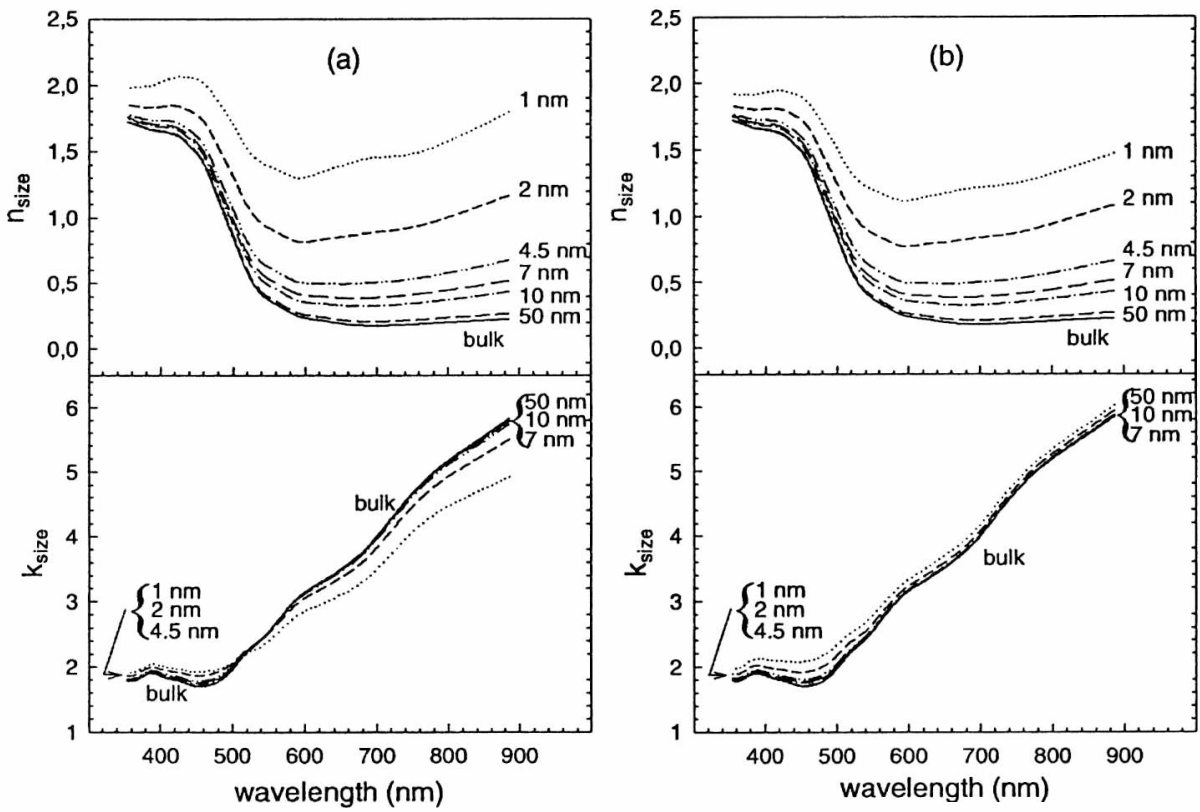

Figure 2. Calculated $n_{\text {size }}$ and $k_{\text {size }}$ as a function of wavelength for different sizes of gold nanoparticles. (a) Values of $\varepsilon^{\prime}$ and $\varepsilon^{\prime \prime}$ are corrected for size. (b) Only $\varepsilon^{\prime \prime}$ is corrected for size while $\varepsilon^{\prime}$ bulk is used for any size.
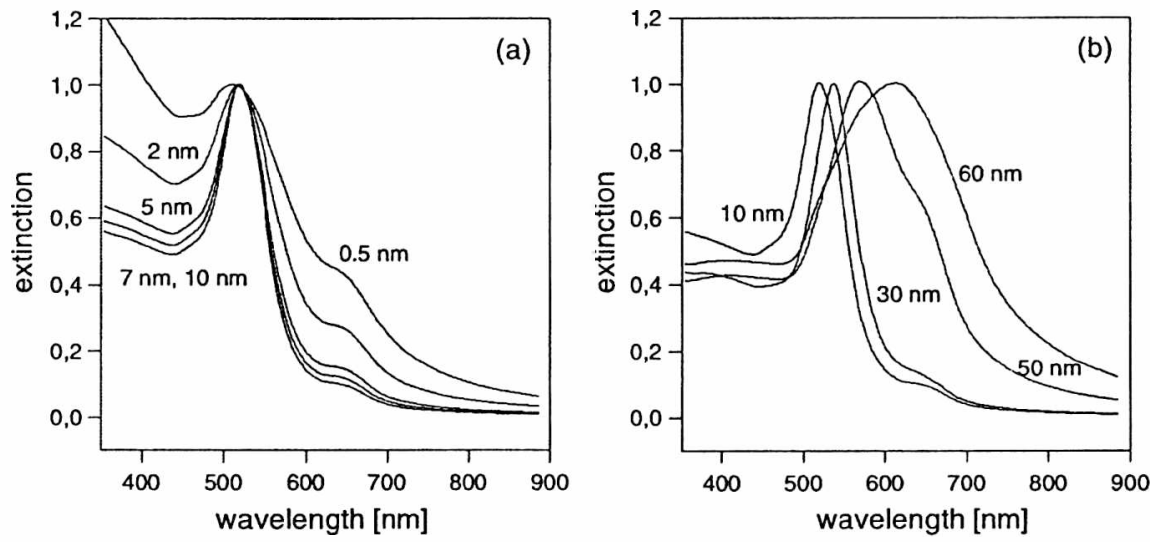

Figure 3. Normalized extinction spectra of gold particles. (a) For small radii the spectral features depend on optical constants calculated with the modification of the electron mean free path. (b) For radii larger than $10 \mathrm{~nm}$, the peak and the width of the spectra are a consequence of the effect of finite size on the Fröhlich resonance.

where the contribution coming from the bound electrons to $\varepsilon^{\prime}$ and $\varepsilon^{\prime \prime}$ were calculated before. Now the values for the real and the imaginary parts of the refractive index corrected by the size, $n_{\text {size }}(\lambda, r)$ and $k_{\text {size }}(\lambda, r)$, can be calculated introducing (5) and (6) in equations (1) and (2):

$$
\begin{aligned}
& n_{\text {size }}(\lambda, r)=\left\{\frac{1}{2}\left[\left(\varepsilon_{\text {size }}^{\prime 2}(\lambda)+\varepsilon_{\text {size }}^{\prime \prime 2}(\lambda, r)\right)^{1 / 2}+\varepsilon_{\text {size }}^{\prime}(\lambda)\right]\right\}^{1 / 2}, \\
& k_{\text {size }}(\lambda, r)=\left\{\frac{1}{2}\left[\left(\varepsilon_{\text {size }}^{\prime 2}(\lambda)+\varepsilon_{\text {size }}^{\prime 2}(\lambda, r)\right)^{1 / 2}-\varepsilon_{\text {size }}^{\prime}(\lambda)\right]\right\}^{1 / 2} .
\end{aligned}
$$

Figure 2(a) shows values for $n_{\text {size }}$ and $k_{\text {size }}$ calculated as a function of wavelength for different sizes of gold nanoparticles. $C=1.1$ was used in this calculation. The choice of this value for parameter $C$ will be discussed in section 4 . It can be seen that $n_{\text {sizc }}$ shows a clear dependence with size up to $10 \mathrm{~nm}$ particle radius, while $k_{\text {size }}$ tends to the bulk values for smaller radius. Special care must be taken to analyse these optical variables in relation to spectral features in the extinction spectra. For metals, the values for $k$ range between 1 and 10 . These are really huge if we recognize that a well absorbing material in the visible has a $k$ value not much greater than $10^{-4}$ [6]. Additionally, it must be considered that the extinction depends on both the $n$ and $k$ values. In figure 2(b) we show the values for $n_{\text {size }}$ and $k_{\text {size }}$ calculated when only the imaginary part of the dielectric function is modified in terms of the size, that is, when $\varepsilon_{\text {size }}^{\prime}$ was taken as $\varepsilon_{\text {bullk. }}^{\prime}$. The reason for this will be discussed later. Mie theory was used to calculate the extinction as a function of the wavelength for different values of the radius. Figure 3 shows the theoretical curves for gold nanoparticles embedded in heptane with radius between 1 and $60 \mathrm{~nm}$. All spectra were normalized to 1 at the wavelength where the maximum of the Fröhlich's resonance occurs.

Figure 3 illustrates the varied optical effects exhibited by small gold metallic particles. In the surface mode region $(r>10 \mathrm{~nm})$, the peak and the width of the absorption spectra depend on the radius of the particle; as the radius increases 


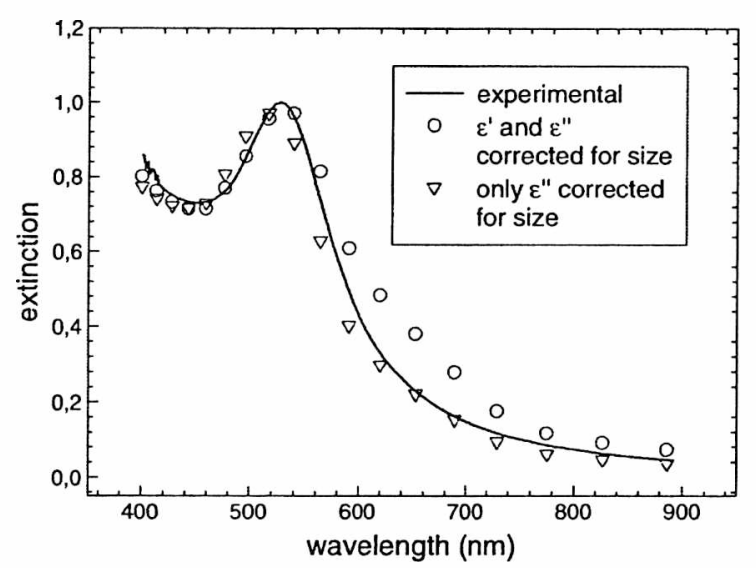

Figure 4. The full curve shows the experimental optical extinction corresponding to the ATS-6 sample as measured with a commercial spectrophotometer. The symbols represent the fitted values (for discrete wavelengths) calculated with Mie theory. Open circles correspond to $\varepsilon^{\prime}$ and $\varepsilon^{\prime \prime}$ corrected for size. Open triangles correspond to only $\varepsilon^{\prime \prime}$ corrected for size. The best fit corresponds to a mean radius of $1.5 \mathrm{~nm}$.

beyond $10 \mathrm{~nm}$, the absorption peak broadens and shifts to longer wavelengths. Absorption spectra by particles with radii between about 2.6 and $10 \mathrm{~nm}$ peak near $520 \mathrm{~nm}$, and they are independent of size. Below $2.6 \mathrm{~nm}$, the spectra again show a dependence with radius: peaks remain near $520 \mathrm{~nm}$ but the contrast between the maximum and the minimum near $440 \mathrm{~nm}$ depends clearly on size. All regions can be explained by Mie theory with optical constants calculated with the modification of the electron mean free path.

\section{Results}

The theoretical extinction curves allow us to perform fits of the spectra corresponding to different samples of gold nanoparticles dispersed in heptane. These curves are calculated for discrete wavelength values for which the refractive index of gold is known from the literature. In figure 4 we show the UV-visible spectra corresponding to sample ATS-6. The theoretical extinction coefficient was calculated with Mie theory for the mean radius of $1.34 \mathrm{~nm}$ determined from the TEM images and optical properties given by equations (7) and (8). The $C$ value was changed to fit the results. The best fit corresponded to $C=1.1$, a value close to that expected from the literature. The fit is good for short wavelengths; however, the calculated values are greater than the experimental values for longer wavelengths. For metals, the real part of the dielectric function evaluated for the bulk is usually used for particles without any modification, while the effect of the mean free path limitation on the imaginary part of the dielectric function is considered [18-20]. When these facts are used to calculate the size-dependent refractive index, the fitting improves. This procedure gives good agreement with the experimental results in gold, but it is not clearly justified. Probably this action hides the size effect on the contribution of the band to band transitions over the dielectric function. This point must be analysed further.

Figure 5 shows the extinction spectrum corresponding to three other samples of gold particles in heptane. For ATS-10

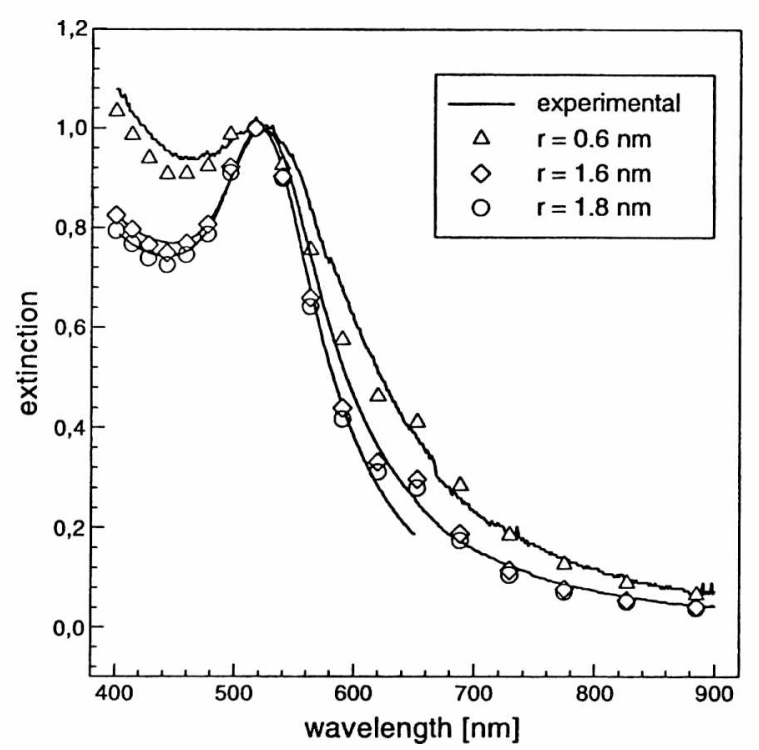

Figure 5. Full curves show experimental optical extinction corresponding to three different gold nanoparticles samples, as measured with a commercial spectrophotometer. The symbols represent the theoretical best fitted values for each case.

(sample with $[\mathrm{ATS}] /\left[\mathrm{Au}^{3+}\right]=10$ ), the mean radius yielded by the TEM image is $1.58 \mathrm{~nm}$, and the best theoretical fit shows a value of $1.6 \mathrm{~nm}$; for ATS-20 (sample with [ATS]/[ $\left.\mathrm{Au}^{3+}\right]=$ 20 ), the TEM image shows $1.96 \mathrm{~nm}$ for the mean radius, while the best theoretical fit yields a value of $1.8 \mathrm{~nm},(8 \%$ difference). The spectrum adjusted with a radius of $0.6 \mathrm{~nm}$ corresponds to a sample produced with another stabilization agent (AES $=3$ (2-aminoethylaminopropyl)trimethoxysilane). This sample (AES-10, with $[\mathrm{AES}] /\left[\mathrm{Au}^{3+}\right]=10$ ) shows a shallow spectrum typical of particles smaller than ATS samples. A similar result was obtained with a sample with $[\mathrm{AES}] /\left[\mathrm{Au}^{3+}\right]=20$; its spectrum, not shown in figure 5, was adjusted with $r=$ $0.7 \mathrm{~nm}$. Unfortunately no TEM images for these AES samples are available; however, particles prepared with the AES stabilization agent must be smaller than the equivalent ATS samples. In addition, for particles prepared with one type of stabilization agent, it is expected that the size of the particles increases with the ratio between the amount of stabilization agent and the concentration of gold used. That is, the radius of the particles must be ordered as ATS-20 > ATS- $10>$ ATS$6>$ AES-20 > AES-10.

The contrast between the maximum and the minimum in the extinction spectra, around 520 and $440 \mathrm{~nm}$ respectively, can be used to evaluate the size of small gold particles. In figure 6 the theoretical contrast for particles with radius between 0.5 and $5 \mathrm{~nm}$ are represented in terms of the size. Good linear dependence can be observed up to $3 \mathrm{~nm}$, but a particle size between 3 and $5 \mathrm{~nm}$ can also be determined by using this plot. Experimental results for the contrast measured on five samples of gold nanoparticles are shown; the radii were determined from the fitting of the whole spectrum as was discussed before. The error bars assume a $15 \%$ error in the radius determination.

\section{Conclusions}

We have shown that Mie theory, combined with a suitably modified dielectric function to include the limitation in 


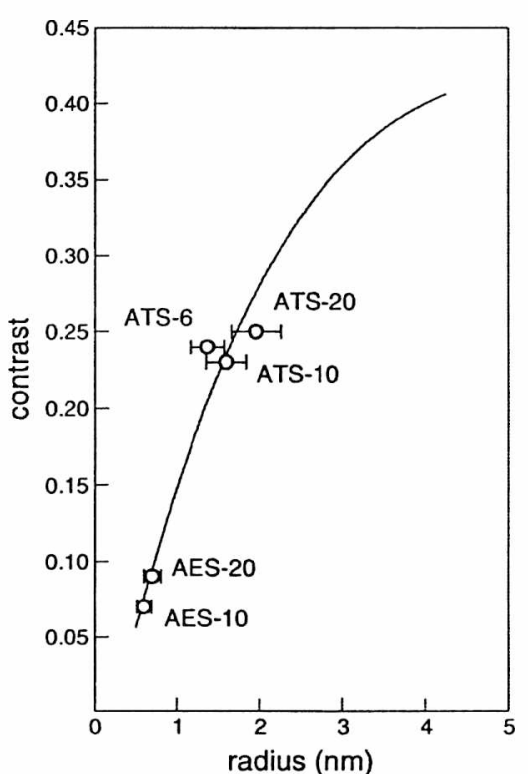

Figure 6. The full curve represents the contrast between the maximum and the minimum in the calculated extinction spectra for gold nanoparticles in terms of their radius. The symbols correspond to the contrast measured in the experimental extinction spectra of AES-10. AES-20, ATS-6, ATS-10 and ATS-20 samples; the radii were assigned from fitting of these spectra (bars assume a $15 \%$ error).

the mean free path of conduction electrons, gives very good agreement with experiments performed with gold nanoparticles. The preparation of these particles assures a nearly spherical form and avoids the formation of clusters. Samples were suspensions of well isolated particles in a low concentration where simple scattering occurs.

This work offers a useful tool to determine the mean radius of gold nanoparticles between 0.5 and $5 \mathrm{~nm}$ from the analysis of the absorbance measurements performed with a commercial spectrophotometer. Sizing can be performed, within a $15 \%$ error, either by fitting the UV-visible spectra or by measuring the contrast between the minimum and the maximum in the $440-520 \mathrm{~nm}$ region. For radii between 5 and $10 \mathrm{~nm}$, optical measurements are not useful for sizing gold particles. Over $10 \mathrm{~nm}$ the extinction spectra show features well correlated with size, and optical extinction spectroscopy is used extensively in sizing metal particles.

For the small particles used in this work there is a very good agreement between the determination of the radius by optical methods and TEM techniques. The relative errors in the determination of the nanoparticles size were smaller than 15\%. Although TEM is the most widely used technique for measuring particle size, it has some disadvantages due to careful sample preparation and the expensive instrument. In such a case, the absorption measurement offers an attractive method to obtain very good results with a low cost.

\section{Acknowledgments}

The authors would like to thank Francisco Jaque-Rechea from Universidad Autónoma de Madrid, Spain, for useful discussions, Raúl Trbojevich from LMC Argentina for the sample preparation and Horacio E Troiani from Texas Material Institute USA for the TEM images. This work was performed with the aid of grants PICT 08852 and PICT 128300 from Agencia Nacional de Promoción Científica y Técnológica, Argentina, and grant X11/324 from Universidad Nacional de La Plata. Support from CYTED network VIIE, Project VIII-9 and Subprograma VIII, Project VIII-2 are also acknowledged. The authors are fellows of CONICET, Argentina.

\section{References}

[1] Jones A R (ed) 2002 Special issue: 6th International Congress on Optical Particle Characterization (Brighton, April 2001); Meas. Sci. Technol. 13 (3) Editorial

[2] Méjean G et al 2003 Towards a supercontinuum-based infrared lidar Appl. Phys. B 77 357.-9

[3] Mejean G, Kasparian J, Yu J, Frey S, Salmon E and Wolf J-P 2004 Remote detection and identification of biological aerosols using a femtosecond terawatt lidar system Appl. Phy's. B 78 535-7

[4] Scaffardi L B, Tocho J O and Yebrin L L 1996 Sizing particles used in the leather industry by light scattering Opt. Eng. 35 $52-6$

[5] Ferri F, Bassini A and Paganini E 1997 Commercial spectrophotometer for particle sizing Appl. Opt. 36 885-91

[6] Bohren C F and Huffman D R 1998 Absorption and Scattering of Light by Snall Particles (New York: Wiley)

[7] Link S and El-Sayed M A 1999 Size and temperature dependence of the plasmon absorption of colloidal gold nanoparticles J. Phys. Chem. B 113 4212-7

[8] Kadono K, Sakaguchi T, Miya M, Matsuoka J, Fukumi T and Tanaka H 1993 Optical non-linear property of Au colloid-doped glass and the laser irradiation stability $J$. Mater. Sci. Mater. Electron. 4 59-61

[9] Brusatin G, Guglielmi M, Innocenzi P, Martucci A and Scarinci G 2000 Materials for photonic applications from sol-gel J. Electroceram 4 151-65

[10] Trbojevich R, Pellegri N, Frattini A, de Sanctis O and Almeida R M 2002 Preparation and isolation of gold nanoparticles coated with a stabilizer and sol-gel compatible agent J. Mater. Res. 17 1973-80

[11] Huglin M B 1972 Light Scattering from Polymer Solutions (New York: Academic)

[12] Palik E D (ed) 1998 Handbook of Optical Constants of Solids vol 1 (New York: Academic) pp 286-95

[13] Granquist C G and Hunderi O 1977 Optical properties of ultrafine gold particles Phys. Rev. B 16 3513-34

[14] Kreibig U and von Fragstein C 1969 The limitation of electron mean free path in small silver particles Z Phys. 224 307-23

[15] Kreibig U 1974 Electronic properties of small silver particles: the optical constants and their temperature dependence $J$. Phys. F: Met. Phys. 4999-1014

[16] Hövel H, Fritz S, Hilger A, Kreibig U and Vollmer M 1993 Width of cluster plasmon resonances: bulk dielectric functions and chemical interface damping Phys. Rev. B 48 $18178-88$

[17] Alvarez M, Khoury J, Schaaff T, Shafigullin M, Vezmar I and Whetten R 1997 Optical absorption spectra of nanocrystal gold molecules J. Phys. Chem. B 101 3706-12

[18] Doremus R, Kao S and García R 1992 Optical absorption of small copper particles and the optical properties of copper Appl. Opt. 31 5773-8

[19] Doremus R H 1964 Optical properties of small gold particles J. Chem. Phys. $402389-96$

[20] Doremus R H 1965 Optical properties of small silver particles J. Chem. Phys. 42 414-7 